This report was prepared as an account of work sponsored by an ageney of the United States Government. Neither the United Siates Government nor any agency thereof, nor any of their employees, makes any warranty, express or inplied, or assumes any legal liability or responsjbility for the accusacy. completeness, or usefulness of any information, appatatus, product, or process disclosed, or represents that its use would nol infringe privately owned rights. Reference herein to any specific commercial preduct, process, of service by trade name, Irademark. mant factuser. or othenwise does not necessarily constitule or inaply its endorsement, recommendation, or favoring by the United States Government or any agency '3ercof. The views and opinions of authors expressed herein do not accessarily state or reflect those of the United States $G$ mment or any agency thereor.

\title{
Propagation of a Gaussian bearn in a nonhomogeneous plasma
}

\author{
E. Mlazzucato \\ Princeton Plasma Physics Laboratory \\ Princeton University \\ Princeton, N. J. 08543
}

\begin{abstract}
The asymptotic theory of Choudhary and Felsen [IEEE Trans. Ant. Prop. AP-21, 827 (1973)] on the propagation of scalar inhomogeneous waves in twodimensional isotropic media is extended to the case of three-dimensional vector fields. The theory is applied to the propagation of Gaussian beams in nonhomogeneous media. The wave trajectory equations are then reformulated for anisotropic media and used for tracking a Gaussian beam in a tokamak plasma.
\end{abstract}

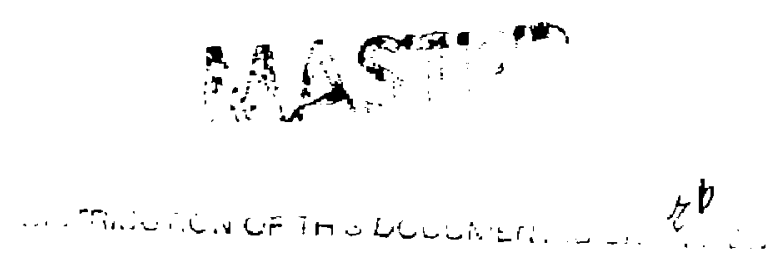




\section{Introduction}

High frequency microwave beams are used in some of the most powerful diagnostics of thermonuclear plasmas. ${ }^{1,2}$ by measuring the spectrum of electromagnetic waves, which are either injected into the plasma or generated within the plasma itself, it is possible to obtain important information on plasma density (interferometry), magnetic field (polarimeiry), electron temperature (radiometry), turbulence (scattering), etc. A crucial factor in the interpretation of experimental data is the location and size of the investigated plasma region. This is determined by the degree of collimation of the probing wave, by the characteristics of the launching and receiving antennas, and by plasma inhomogeneities which deflect and spread the wave beam. To improve the measurement spatial localization, use is made very often of highly collimated waves, such as the Gaussian beam of a laser, and waves are launched and received with focussing elements, like lenses and curved mirrors. The effect of plasma refraction is estimated with ray-tracing techniques based on the theory of geometrical optics. ${ }^{3,4}$ Cnfortunately, even though the wavelength of the probing wave is, in most cases, much shorter than the plasma scale lengths, the approximation of geometrical optics breaks down near the focal region where the diagnosed plasma is usually located.

In the conventional theory of geometrical optics, ${ }^{3,4}$ the local description of the neld is given in terms of homogeneous plane waves $\mathbf{E}(\mathbf{r}) \exp [-i k S(\mathbf{r})]$, where $k$ is the free-space wave number, and $\mathbf{E}(\mathbf{r})$ is a spatially dependent amplitude. The function $S(\mathbf{r})$ is the wave phase which is assumed to be a real quantity in a loss-free medium. These functions are obtained from the leading terms of an asymptotic series expansion of the solution of Maxwell's equations and can only describe fields whose armplitude does not change appreciably over a distance of a wavelength. This impairs the validity of the geometrical approximation near a focal point or a caustic. In order to deal with these cases, Choudhary and Felsen ${ }^{5}$ have extended the theary of geometrical optics of two-dimensional scalar fields by including evanescent or inhomogeneous waves, i.e., plane waves with complex phase (or eikonal), $S(\mathbf{r})$.

In this paper, the theory of geometrical optics with complex eikonal is used 
for the study of wave beam propagation in nenhomogeneous media. In Sec. II, the existing scalar theory ${ }^{5}$ is reviewed and extended to three-dimensional vector fields. In Sec. III, the theory is applied to the propagation of Gaussian beams in nonhomogeneous isotropic media. In Sec. IV, the equations of the wave trajectory are generalized for anisotropic media, and the results are used for the propagation of a Gaussian beam in a tokamak plasma. Finally, the conclusions are summarized in Sec. $V$.

\section{Complex Eikonal}

The formulation of geometrical optics with evanescent waves follows the route of conventional theory. The only difference is that the local plane waves are assumed to have a complex rather than a real phase, $S(\mathbf{r})$. The electric field is taken in the form

$$
\mathbf{E}(\mathbf{r}, t)=\mathbf{E}_{\mathrm{o}}(\mathbf{r}) \exp [-i k S(\mathbf{r})+i \omega t],
$$

where $\omega$ is a real frequency, and $\mathbf{E}_{\circ}(\mathbf{r})$ is a slowly varying function which, as an Ansatz, is expressed as a series in prwers of $1 / k$

$$
\mathbf{E}_{\mathrm{o}}(\mathbf{r})=\sum_{n \geq 0} \frac{\mathbf{e}_{\mathbf{n}}}{k^{n}},
$$

where $e_{n}$ are complex functions of position. A priori, with very few simple exceptions, it is impossible to say whether this expansion is valid either as a convergent or as an asymptotic series. Nevertheless, experience supports its use and, in most cases, it is the orly tractable method.

Substituting (1) and (2) and equivalent equations for the magnetic field into Maxwell's equations, one obtains from the lowest order terms the eikonal equation

$$
(\nabla S)^{2}=n^{2}
$$

and from the first order terms the transport equation for the amplitude $e_{0}$

$$
\left(\nabla^{2} S+2 \nabla S \cdot \nabla\right) \mathrm{e}_{\mathrm{o}}+2\left(\mathrm{e}_{\mathrm{o}} \cdot \nabla n / n\right) \nabla S=0 .
$$


The vector $e_{0}$ must also satisfy the subsidiary condition

$$
\mathbf{e}_{\mathrm{o}} \cdot \nabla S=0
$$

which is derived from the lowest order term in the divergence of the electric displacement. The function $n(\mathbf{r})$ which appears in Eq. (3) and (4) is the local refractive index of the medium which, for simplicity, is assumed isotropic and loss-free.

By casting $e_{0}$ in the form

$$
\mathbf{e}_{\mathrm{o}}=E(\mathbf{r}) \mathbf{e}
$$

with $E(\mathbf{r})$ a solution of

$$
\left(\nabla^{2} S+2 \nabla S \cdot \nabla\right) E=0
$$

the amplitude transport equation (4) becomes

$$
\nabla S \cdot \nabla \mathbf{e}+(\mathbf{e} \cdot \nabla n / n) \nabla S=0
$$

with the auxiliary condition

$$
\text { e. } \nabla S=0
$$

To solve the eikonal equation, it is convenient to write $S(\mathbf{r})=K(\mathbf{r})+i I(\mathbf{r})$, with $R(\mathbf{r})$ and $I(\mathbf{r})$ real functions, and to introduce the unit vectors

$$
\mathrm{s}=\nabla R / \beta, \quad \mathrm{t}=\nabla I / \alpha
$$

with $\beta=|\nabla R|$ and $\alpha=|\nabla I|$. Upon separation into real and imaginary parts, Eq. (3) gives

$$
\begin{aligned}
\beta^{2}-\alpha^{2} & =n^{2} \\
\mathbf{s} \cdot \mathbf{t} & =\mathbf{0}
\end{aligned}
$$

Along the tangent lines of the vector field s, which are normal to the surfaces of constant phase $R$, the imaginary part of $S$ remains constant. These lines are the 
equivalent of the phase rays of conventional geometrical optics. The important difference is that, in the case of complex eikonals, the power flow deviates from the direction of advancing phase fronts..$^{5}$. Along the tangent lines of the vector field $\mathbf{t}$, which are normal to the surfaces of constant exponential amplitude $I$, the real part of $S$ remains constant. In the following, these lin ; will be referred as phase and amplitude paths (or rays), respectively. Nevertheless, it should be stressed that neither the total wave amplitude nor the total phase is constant along these lines. If $s$ and $t$ are the arc-length along these two sets of lines, the trajectory equations are

$$
\frac{d}{d s}(\beta s)=\nabla \beta, \quad \frac{d}{d t}(\alpha t)=\nabla \alpha,
$$

with $\beta$ and $\alpha$ subject to condition (10).

By kriting

$$
E(\mathbf{r})=\exp [u(\mathbf{r})+i v(\mathbf{r})],
$$

with $u$ and $v$ real functions, Eq. (7) reduces to the following set of equations

$$
\begin{aligned}
& \nabla \cdot(\beta \mathbf{s})+2 \beta \mathbf{s} \cdot \nabla u-2 \alpha \mathbf{t} \cdot \nabla v=0, \\
& \nabla \cdot(\alpha \mathbf{t})+2 \beta \mathbf{s} \cdot \nabla v+2 \alpha \mathbf{t} \cdot \nabla u=0 .
\end{aligned}
$$

These equations were first derived in Ref. 4 as the transport equations for twodimensional scalar fields. In the case of vector fields, the solution of Eq. (8) is also needed for determining the wave amplitude.

To solve Eq. (8), it is convenient to introduce the unit vector $\phi=\mathbf{s} \times \mathbf{t}$ and write $\mathbf{e}=e_{s} \mathrm{~s}+e_{t} \mathrm{t}+e_{\phi} \phi$ which put condition (9) into the form

$$
\beta e_{i}+i \alpha e_{t}=0 \text {. }
$$

This equation, together with (10), (11), and (12), makes the component of Eq. (8) along the $s$-axis equivalent to the component along the $t$-axis. In other words, once condition (15) is verified at one point of a phase or amplitude path, it remains valid over the whole path. Thus the field $e$ is determined by Eq. (15) and the $t$ - and $\phi$-components of Eq. (8) which can be written as

$$
\nabla_{\cdot}\left(\frac{n}{\beta} e_{t}\right)+i\left(\frac{\alpha}{\beta}\right) \nabla_{t}\left(\frac{n}{\beta} e_{t}\right)=e_{\phi}\left[i\left(\frac{\beta}{n}\right) \nabla_{\phi}\left(\frac{\alpha}{\beta}\right)+\frac{n}{\beta} \phi \cdot \nabla_{\imath} t\right],
$$


and

$$
\nabla_{s} e_{\phi}+i\left(\frac{\alpha}{\beta}\right) \nabla_{t} e_{\phi}=-\frac{n}{\beta} e_{t}\left[i\left(\frac{\beta}{n}\right) \nabla_{\phi}\left(\frac{\alpha}{\beta}\right)+\frac{n}{\beta} \phi \cdot \nabla_{\Delta} t\right]
$$

where, for brevity, $\nabla_{s} \equiv \mathbf{s} \cdot \nabla, \nabla_{t} \equiv \mathbf{t} \cdot \nabla$ and $\nabla_{\phi} \equiv \phi \cdot \nabla$. These equations were obtained by using Eqs. (10), (11), (12), and the relation

$$
\phi \cdot\left(\frac{\partial t}{\partial s}+\frac{\partial s}{\partial t}\right)=0
$$

which is derived from the vector identity

$$
\nabla(\mathbf{a} \cdot \mathbf{b})=\mathbf{a} \times(\nabla \times \mathbf{b})+\mathbf{b} \times(\nabla \times \mathbf{a})+(\mathbf{a} \cdot \nabla) \mathbf{b}+(\mathbf{b} \cdot \nabla) \mathbf{a}
$$

by using $\mathbf{a}=\nabla R, \mathrm{~b}=\nabla I$, and Eq. (11).

From (16) and (17) one obtains the equation

$$
\nabla, f+i\left(\frac{\alpha}{\beta}\right) \nabla_{t} f=0,
$$

with $f=\left(n e_{l} / \beta\right)^{2}+e_{\phi}^{2}$, which admits solutions with $f=c o n s t$. along phase and amplitude paths. For the case of axial symmetry, like a circular Gaussian beam propagating in vacuum or in a cylindrical lens-like medium, the right-hand side of Eqs. (16) and (17) are zero and the functions $(n / \beta) e_{t}$ and $e_{\phi}$ can be taken constant along a phase or an amplitude path.

This completes the set of equations for the lowest order approximation of the propagation of evanescent waves in isotropic media.

\section{Gaussian beam}

In this Section, the theory described in Sec. II is applied to the study of the propagation of a Gaussian bearn. The computation of the wave field proceeds as follows:

(a) Solve the phase path equation (12) subject to conditions (10) and (11), given an initial phase front where the values of $R(=$ comst.) and $I(r)$ are prescribed. This provides the functions $\alpha(\mathbf{r})$ and $\beta(\mathbf{r})$ and the field of vectors $\mathbf{s}, \mathbf{t}$, and $\phi$. 
(b) Solve the amplitude transport equations (13) and (14), given the value of $E(\mathbf{r})$ on the initial phase front.

(c) Solve the amplitude transport equations (16) and (17) together with Eq. (15), given the value of $e$ on the initial phase front.

The phase path equation can be integrated with the Runge-Kutta method, ${ }^{6}$ while the two-step Lax-Wendroff scheme ${ }^{6}$ can be used for solving the amplitude transport equations.

To solve the field equations, it is convenient to define on the initial phase surface a system of curvilinear coordinates $(\rho, \phi)$ with the contours of constant amplitude $I$ as the $\phi$-lines. If $\rho$ and $\phi$ are the unit vectors along the two coordinate lines, $\phi$ coincides with the homonymous vector of Sec. II. By using the solution of the phase equation (12), which can be written as

$$
\nabla \mathrm{s}=\frac{\nabla_{\perp} \beta}{\beta},
$$

with $\nabla_{\perp}=\nabla-\nabla_{\iota}$, it is possible to extend the definition of these coordinates to every phase surface by assuming constant values of $\rho$ and $\phi$ on a phase path. Since the function $I(\mathbf{r})$ is also constant along these rays, the vector $\phi$ of Sec. II continues to coincide with the unit tangent vector of the $\phi$-line. By making use of the equation

$$
\nabla_{t}=\frac{1}{(\rho \cdot t)}\left(\nabla_{\rho}-(\rho \cdot \phi) \nabla_{\phi}\right),
$$

with $\nabla_{\rho} \equiv \rho \cdot \nabla$, one can rewrite the transport equations in the new system of coordinates $(\rho, \phi, s)$.

Before dealing with the case of nonhomogeneous media, it is instructive to consider that of vacuum propagation and compare the results with known analytic solutions of the wave equation. Figure 1 shows the phase paths obtained with numerical integration of $\mathrm{Eq}$. (19) for a circular beam propagating in vacuum with a wavelength of $0.5 \mathrm{~cm}$. The wave has an initial spherical phase front with a radius of curvature of $150 \mathrm{~cm}$ and a Gaussian electric field distribution with $1 / e$ radius of $10 \mathrm{~cm}$. Obviously, in the conventional theory of geometrical optics all rays would have converged towards a single point. 
These results and those obtained from the amplitude transport equations can be compared with well-known and widely used solutions of the parabolic wave equation. ${ }^{7}$ In a Cartesian coordinate system $(x, y, z)$ with the $z$-axis along the direction of beam propagation and the vector potential $\mathbf{A}$ polarized along the $x$-axis, a solution of the parabolic wave equation is ${ }^{8}$

$$
\mathbf{A}=\mathbf{x} U \exp \left[-i k z-\left(x^{2}+y^{2}\right) / w^{2}\right],
$$

with

$$
U=w^{-1} \exp \left[i \psi(z)-i k\left(x^{2}+y^{2}\right) / 2 R\right],
$$

and $w^{2}(z)=(2 b / k)\left(1+z^{2} / b^{2}\right), 1 / R(z)=z /\left(z^{2}+b^{2}\right)$, and $\tan [\psi(z)]=z / b$. The argument of the exponential in Eq. (21) must be compared with the eikonal $S(\mathbf{r})$, the phase $\psi(z)$ of Eq. (22) with the value of the function $v(\mathbf{r})$ obtained from the solution of Eqs. (13) and (14), and the value of $R$ with the radius of curvature of the phase fronts. Excellent agreement is found in all three cases; consequently, the theory of Sec. II is equivalent to the parabolic approximation. Since this consists of neglecting the second derivative in $z$ of the slowly varying wave amplitude, Eq. (21) implies that the approximation is valid whenever $(k w)^{-2} \ll 1$, i.e., when the beam radius is larger than the wavelength. This condition can be rewritten as $\alpha^{2}<<1 \approx \beta^{2}$.

Let us now consider the case of propagation in nonhomogeneous media. Figure 2 shows the effect of a variable index of refraction with spatial dependence $n(\mathbf{r})=1-0.3 \exp \left[-\left((x-20)^{2}+(z-25)^{2}\right) / 75^{2}\right]$ on a Gaussian beam with initial linear polarization and the same conditions of Fig. 1. As an example of solution of the amplitude transport equations, Fig. 3 displays the value of $e_{t}$ versus the optical length $d=\int_{0}^{*} 3\left(s^{\prime}\right) d s^{\prime}$ for the rays of Fig. 2 which propagate below the central ray. The standard theory of geometrical optics would have given constant values along completely different trajectories.

\section{Wave tracking in anisotropic media}

The theory described in Sec. II cannot be used for tracking a wave in an arisotropic medium which is the case of interest for the diagnostic applications mentioned in the Introduction. 
The starting point in the derivation of the wave trajectory equations in anisotropic media is again the eikonal equation, i.e., the consistency condition for solution of the lowest order eikonal approximation of Maxwell's equations. As in Sec. II, we consider only the case of a loss-free medium and, analogously to Eq. (3), we cast the eikonal condition in the form

$$
D(\mathbf{r}, \nabla S) \equiv(\nabla S)^{2}-n^{2}(\mathbf{r}, \nabla S)=0
$$

where now, contrary to the isotropic case, the refractive index $n=n(r, \nabla S)$ is also a function of the complex vector $\nabla S=\beta s+i a t$. In the following, we shall consider the case of a cold magnetized plasma and take for $\boldsymbol{n}$ the Appleton-Hartree expression. ${ }^{9}$ This depends also on the mode of propagation, i.e., the ordinary and the extraordinary modes.

The theory is considerably simplified by the assumption that $\alpha<<\beta$. By expanding the function $D(\mathbf{r}, \nabla S)$ around $\nabla S=\beta$ s and keeping terms of up to the second order in $(\alpha / \beta)$, we get

$$
D(\mathbf{r}, \boldsymbol{\beta})+i \alpha(\mathbf{r}) \mathbf{t} \cdot \frac{\partial D(\mathbf{r}, \boldsymbol{\beta})}{\partial \boldsymbol{\beta}}-\frac{\alpha^{2}(\mathbf{r})}{2} \mathbf{t} \mathbf{t}: \frac{\partial^{2} D(\mathbf{r}, \boldsymbol{\beta})}{\partial \boldsymbol{\beta} \partial \boldsymbol{\beta}}=0
$$

where $\beta=\beta$ s, and use is made of the dyadic notation. Upon separation into real and imaginary parts, Eq. (24) gives for a loss-free medium (i.e., when $n^{2}(r, \beta)$ is a real function)

$$
H(\mathbf{r}, \boldsymbol{\beta}) \equiv \beta^{2}-n^{2}(\mathbf{r}, \boldsymbol{\beta})-\alpha^{2}(\mathbf{r})+\frac{\alpha^{2}(\mathbf{r})}{2} \mathrm{tt}: \frac{\partial^{2} n^{2}(\mathbf{r}, \beta)}{\partial \boldsymbol{\beta} \partial \boldsymbol{\beta}}=0
$$

and

$$
\mathbf{t} \cdot\left(2 \boldsymbol{\beta}-\frac{\partial n^{2}(\mathbf{r}, \boldsymbol{\beta})}{\partial \boldsymbol{\beta}}\right)=0
$$

For an isotropic medium (i.e., when $n^{2}=n^{2}(\mathbf{r})$ ), these two equations coincide with Eqs. (10) and (11), respectively.

From Eq. (25) one can derive the wave trajectory equations which, in Hamiltonian form, are

$$
\frac{\boldsymbol{d}}{d l}=\frac{\partial H / \partial \boldsymbol{\beta}}{|\partial H / \partial \boldsymbol{\beta}|}, \frac{d \boldsymbol{\beta}}{d l}=-\frac{\partial H / \partial \mathbf{r}}{|\partial H / \partial \boldsymbol{\beta}|}
$$


where $l$ is the arc-length along the trajectory. Equations (27) must be solved self-consistently with Eq. (26). The latter constrains the group velocity to be tangent to the surface of constant exponential amplitude $I$ which, contrary to the case of isotropy, is not necessarily orthogonal to the surface of constant phase $R$ (i.e., $\mathbf{s} \cdot \mathbf{t} \neq 0$ ).

For an anisotropic medium, the derivation of the transport equations for the lowest order field amplitudes is considerably more difficult than in the case of isotropy. The problem is the same as in the standard theory of geometric optics in media with non-Hermitian dielectric tensors which has been treated very elegantly in Ref. 10. Since the algebra quickly becomes very cumbersome, the reader is referred directly to this paper, and to Ref. 11 for more algebraic details.

Figures 4 and 5 show two examples of numerical integration of the trajectories equations for the case of a Gaussian beam propagating in a tokamak medium with the extraordinary and the ordinary mode, respectively. The beam characteristics and its initial conditions are those of Fig. 1, and, like in many of the diagnostics mentioned in the Introduction, it is injected from the top of the plasma torus along a downward vertical direction. The magnetic configuration has a Shafranov equilibrium ${ }^{12}$ with the characteristics of a typical TFTR discharge in the enhanced confinement mode, ${ }^{13}$ and with a toroidal magnetic field of $50 \mathrm{kG}$. The electron density is constant on magnetic surfaces with the value of $7 \times 10^{13} \mathrm{~cm}^{-3}$ on the magnetic axis.

It is the presence of the last two terms in the left-hand side of Eq. (25) which prevents the formation of a focal point or a caustic surface. Since the contribution of these terms becomes important only near the focal region, one must switch to the new formalism only in the vicinity of the focus. Nevertheless, it is less cumbersome to use everywhere the wave trajectory equations (27) since their numerical complexity is not greater than that of the ray equations of conventional geometrical optics. As a matter of fact, the numerical code used for tracking the beams of Figs. 4 and 5 was just a straightforward modification of a standard ray-tracing code used previously ${ }^{14}$ for the study of Electron Cyclotron Heating in tokamaks. 


\section{Conclusion}

In this paper, the asymptotic theory of Choudhary and Felsen on the propagation of scalar inhomogeneous waves in two-dimensional isotropic media has been extended to three-dimensional vector fields. The theory has been applied to the propagation of Gaussian beams in vacuum, where it is in very good agreement with analytic solutions of the parabolic wave equation, and in nonhomogeneous media.

The wave trajectory equations have been generalized for anisotropic media by expanding the eikonal equation into a power series of $(\alpha / \beta)$ and keeping terms of up to the second order. In a loss-free medium, the solutions of these equations are subject to the constraint that the direction in which the amplitude decays most rapidly is perpendicular to the wave trajectory. An example of solution has been given for the case of propagation of a Gaussian beam in a tokamak plasma.

In conclusion, the geometrical theory of propagation of inhomogeneous waves in nonuniform media has been extended to vector fields and to anisotropic media. It provides a simple and powerful tool for calculating the lowest order diffaction effects in complicated nonhomogeneous media where the solution of the wave equation becomes very cumbersome, if not impossible.

\section{Acknowledgments}

The author vishes to express thanks to Dr. P. L. Colestock, Dr. I. Fidone, and Dr. C. K. Phillips for fruitful discussions.

This work was supported by U.S. Department of Energy Contract No. DEAC02-76-CHO-3073. 


\section{References}

1 M. A. Heald and C. B. Wharton, Plasma Diagnostics with Microwaves (Wiley, New York, 1965).

2 G. Bekefi, Radiation Processes in Plasmas (Wiley, New York, 1966).

3 M. Born and E. Wolf, Principles of Optics (Pergamon, Oxford, 1964).

4 M. Kline and I. W. Kay, Electromagnetic Theory and Geometrical Optics (Interscience, New York, 1965).

5 S. Choudhary and L. B. Felsen, IEEE Trans, Antennas Propag. AP-21, 827 (1973).

6 W. H. Press, B. P. Flannery, S. A. Teukolsky, and W. T. Vetterling, Numerical Recipes (Cambridge University Press, Cambridge, 1988).

7 V. A. Fock, Electromagnetic Diffaction and Propagation Problems (Pergar mon, New York, 1965).

8 H. Kogelnik, Appl. Opt. 4, 1562 (1965).

9 T. H. Stix, The Theory of Plasma Waves (McGraw-Hill, New York, 1962).

10 L. Friedland and I. Bernstein, Phys. Rev. A 22, 1680 (1980).

11 I. Fidone, G. Granata, and R. L. Meyer, Phys. Fluids 25, 2249 (1982).

12 V. D. Shafranov, in Revieus of Plasma Physics, edited by M. A. Leontovich (Consultants Bureau, New York, 1966), Vol. 2, p.103.

13 J. D. Strachan, M. Bitter, A. T. Ramsey, M. C. Zarnstorf, V. Arunasalam, M. G. Bell, N. L. Bretz, R. Budny, C. E. Bush, S. L. Davis, H. F. Dylla, P. C. Efthimion, R. J. Fonck, E. Fredrickson, H. P. Furth, R. J. Goldston, L. R. Grisham, B. Grek, R. J. Hawryluk, W. W. Heidbrink, H. W. Hendel, K. W. Hill, H. Hsuan, K. P. Jaehnig, D. L. Jassby, F. Jobes, D. W. Johnson, L. C. Johnson, R. Kaita, J. Kamperschroer, R. Z. Knize, T. Kozub, H. Kugel, B. LeBlanc, F. Levinton, P. H. La Marche, D. M. Manos, D. K. Mansfield, K. McGuire, D. H. McNeill, D. M. Meade, S. S. Medley, W. Morris, D. Mueller, E. B. Nieschmidt, D. K. Owens, H. Park, J. Schivell, G. Schilling, G. L. Schmidt, S. D. Scott, S. Sesnic, J. C. Sinnis, F. J. Stauffer, B. C. Stratton, 
G. D. Tait, G. Taylor, H. H. Towner, M. Ulrickson, S. von Coeler, R Wieland, M. D. Williams, K-L. Wong, S. Yoshikawa, K. M. Young; and S. J. Zueben, Phys. Rev. Lett. 58, 1104 (1987).

14 E. Mazzucato, I. Fidone, and G. Granata, Phys. Fluids 30, 3745 (1987). 


\section{Figure Captions}

Fig. 1. Phase paths of a Gaussian beam propagating in vacuum along the $z$-axis with a wavelength of $0.5 \mathrm{~cm}$, starting from an initial spherical phase front with a radius of curvature of $150 \mathrm{~cm}$.

Fig. 2. Same as Fig. 1 with a variable index of refraction.

Fig. 3. Value of $e_{t}$ versus the optical length $d=\int_{0}^{s} \beta\left(s^{\prime}\right) d s^{\prime}$ along the phase paths of Fig. 2 which propagate below the beam central ray. Values increase from the center to the edge of the beam.

Fig. 4. Wave trajectories of the Gaussian beam of Fig. 1 propagating in a tokamak plasma with the extraordinary mode. The plasma density is assumed constant on magnetic surfaces (dashed circles).

Fig. 5. Same as Fig. 4 with the ordinary mode. 


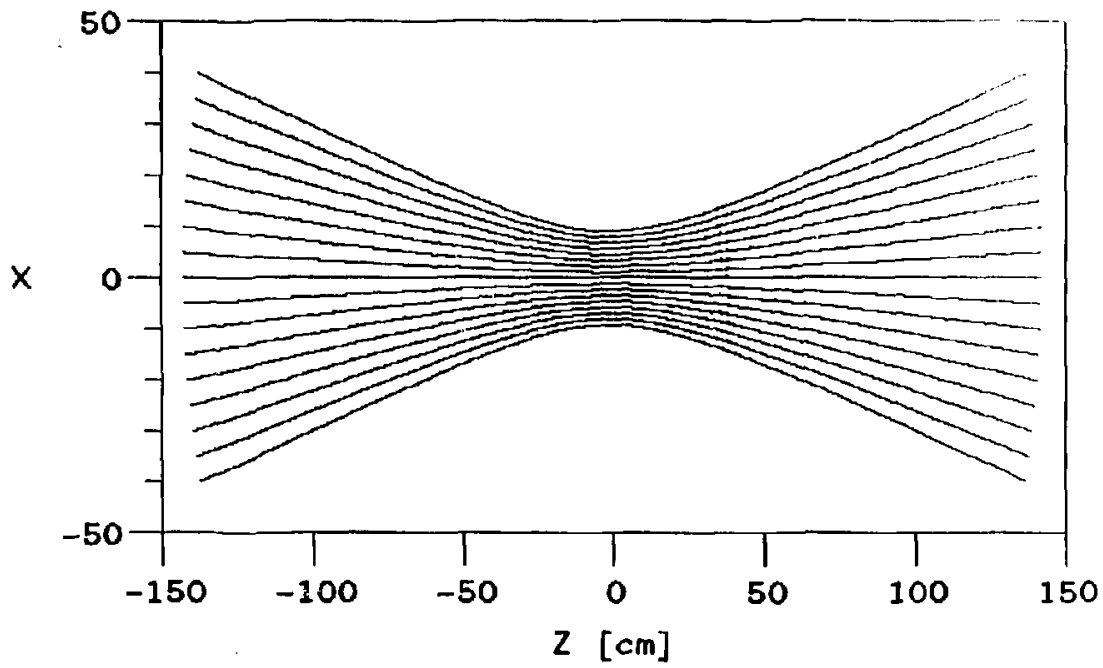

Fig. 1 


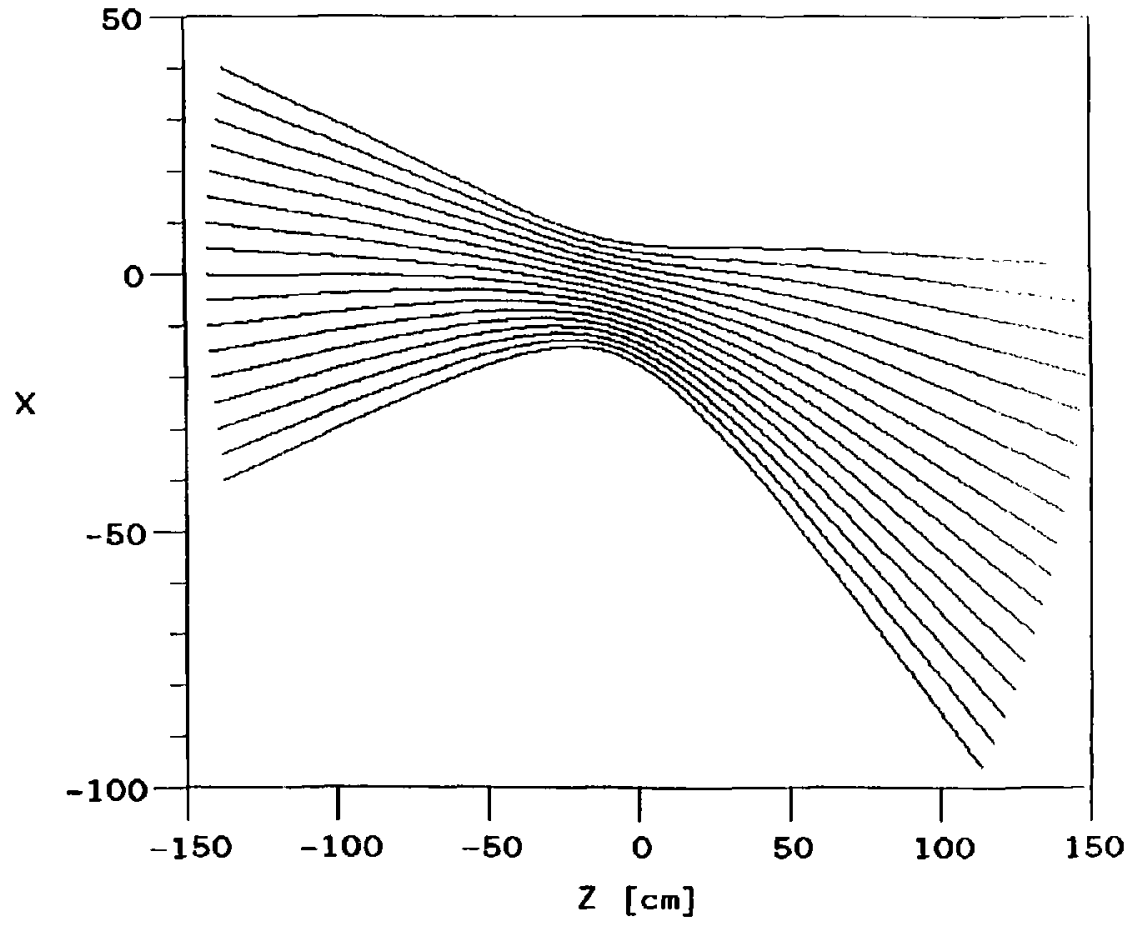

Fig. 2 


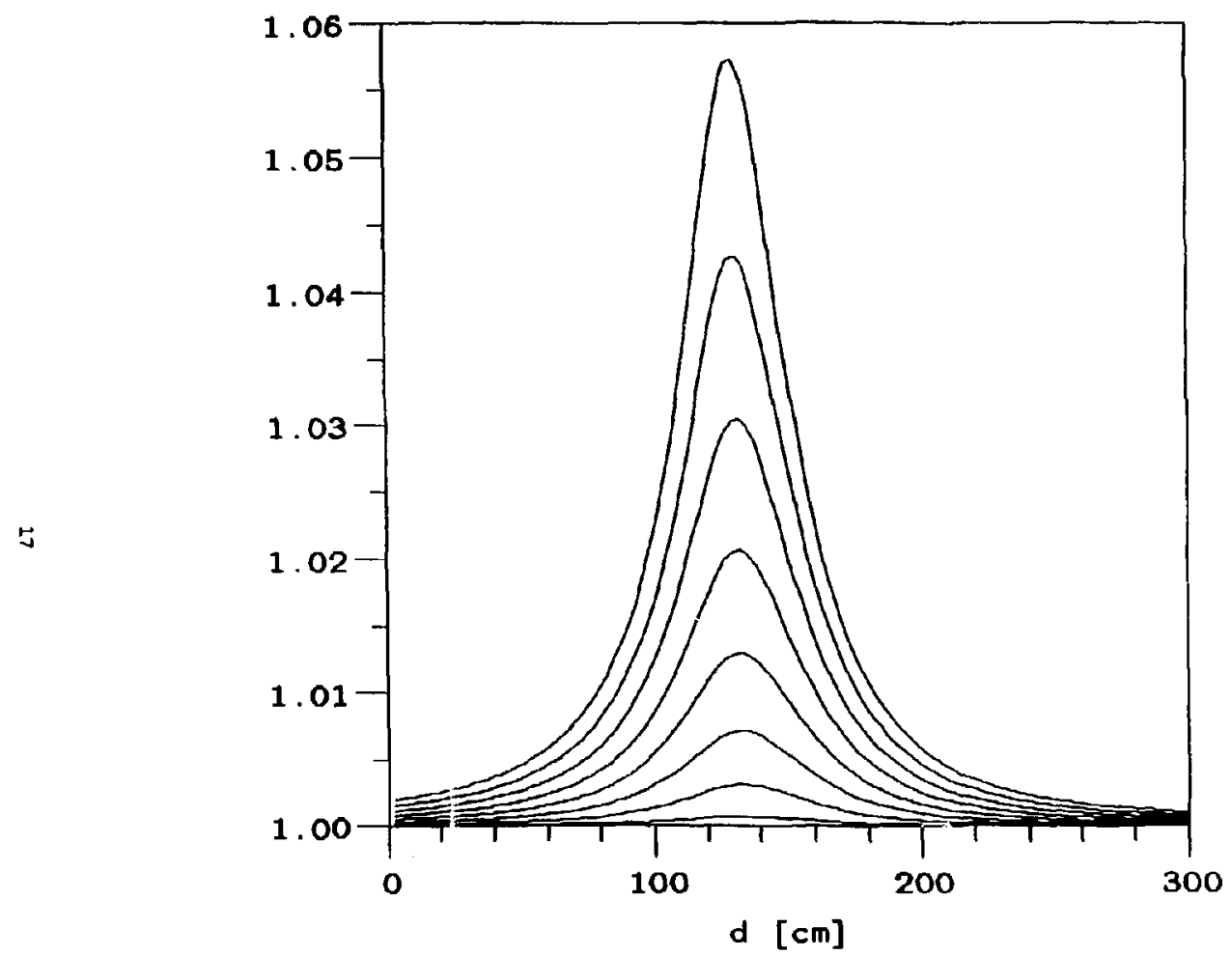

Fig. 3 


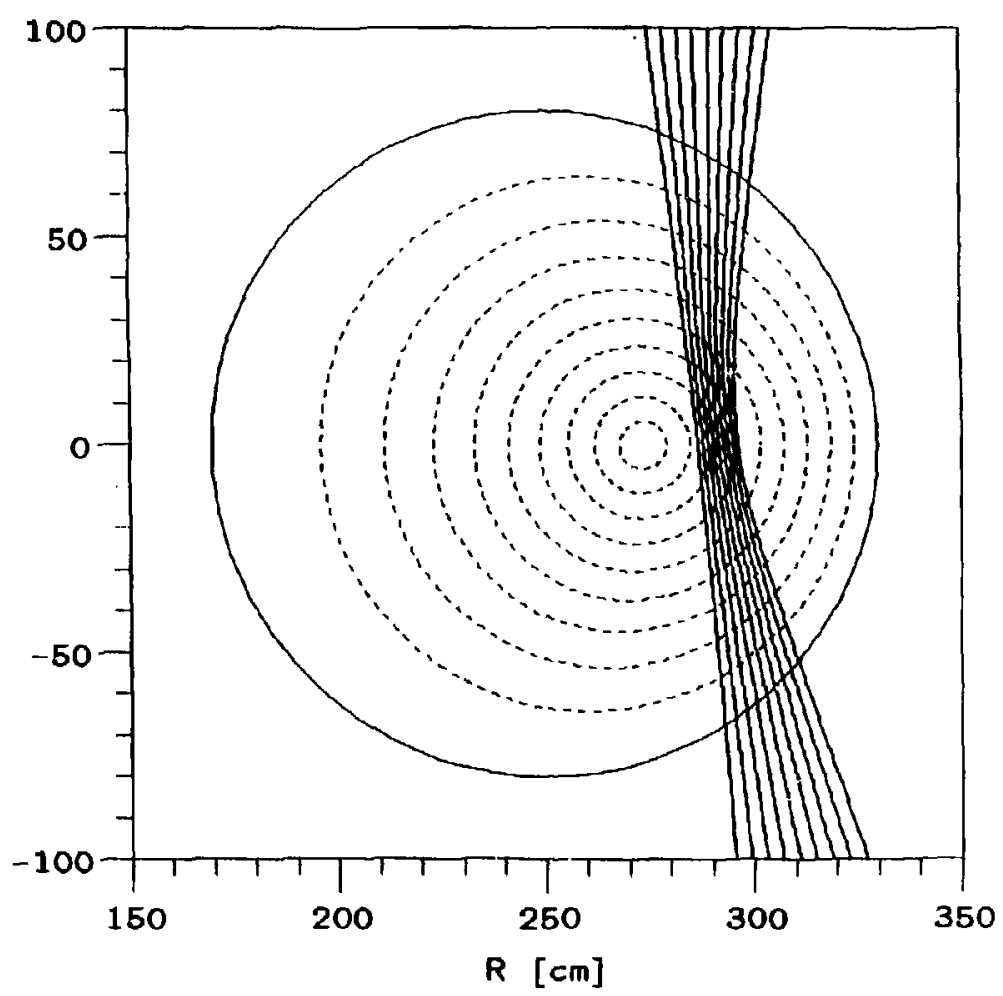

Fig. 4 


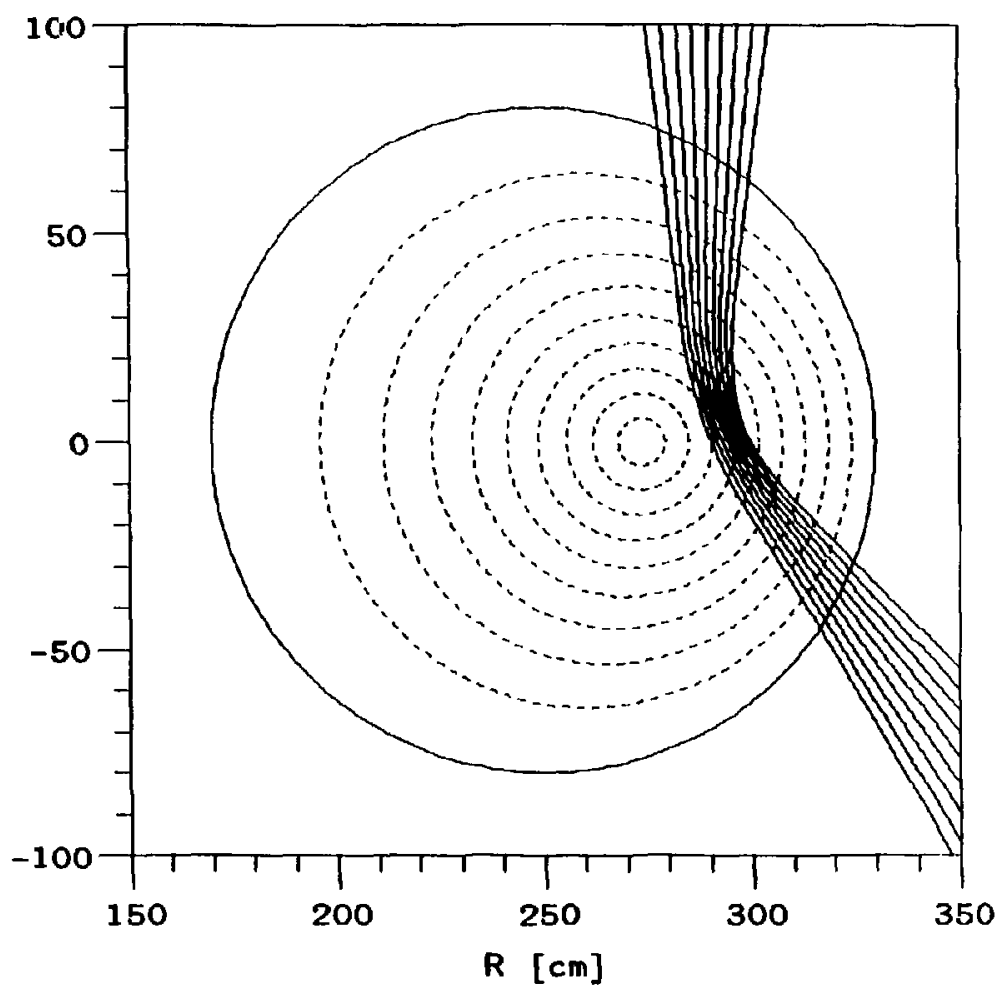

Fig. 5 
Or. Frank d. Paoloni. Univ of wollongong, AUSTRAL I Prot. M.H. Brennen, Unir Sydner, Australia

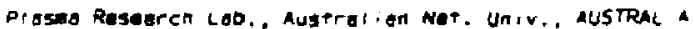
Prot. I,R. Jones, Flinders Univ., austantia Prot. F. Cab, Inst Theo Phys, austria

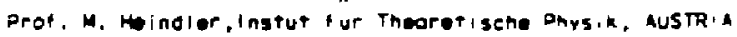
M. Coossens, Astronaisen ingrituut, BELGIN Ecole Royele Militalre, tob de Phys P. asmas, BElgilu Comission-European, DoProt. R. Bouciaue, Rijksuniversiteit Gant, BELGium Dr. P.H. Sakanaka, Instituto F isica, BRhZi Instituto Do Pesquigas Espoc i OSI=tNPE, ERAZ'L Documants Office. Atocic Energr of Canada . inted. CANADA Dr. M.P. Bechynsti, wo Teennologies, Inc., CakaOh Dr. H.M. Skersgara, University of Saskatchoman. ColudoA Dr. H. Bernora. Universiry of British Columbie. Canaba Prof. t. Taichenn, Univ. of montras! , carada Prot. S.R. Sromivaten, University of Calgary, chupa Prot. Tucor W. Johnston, IMB-Energie. CAknoA Dr. Botton. Centre cantion de fusion magnetigue. Camada Or. C.R. Jems, Univ, of Alberto, CANAD Dr. Poter Lukac, Komensono uniy, CzEckoslovaxia The Librarion, Culhe Laboretory, ENGLAND The Librarian, Rutharford Agoietan Laboratory, ENGLAND Mrs. S.A. Hutchinson, JET L ibrgry, EHCLAND C. Houttat, Lob. de Phrsique os Milieur lonisés, FRarce J. Radet, EEN/CHDARADE - Bet 50s, FRAnce 4. C. Rinni, Librarion, Univ. of laannine, GREECE Or. To mul, Acedery Bibliographic Ser. . Mowg kong Dreprint Library. Hungorien Acesery of Seiences, HuMrahy Dr. B. Das Gupte, Sahe inst of Muel. Phys.. INDia Dr. P. Kas, Institute for Plases Research, Noik Or. Phi I ip Rosentiu, Isrest Inst. of Teen, I SRAEL Liorarian, Int'I Ctr Theo Phys, ITALY Prot, G. Rostagni, istituto Ge lonizzat, 2, CAA, ITALY Miss Clelia Do Palo, Assoc étRATOH-EHEA, ITALT Dr. G. Grosero. Istituto di Fisica al Plasea, ITALT or. H. remero. Tosniba Ras 8 Dav, JAPAN
Pro*, Kgegkbmi, Mtomic Energy Ros. Institura, , APaN Prot. Kroj, Nishimana, Jnis of w roshima, JAPAN

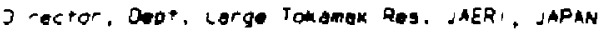
P-op. Satosh, Iton. Mrushy Univarsitr, JAPAN Pestaren into Conear. Nagove univers ty, JAPAN Prol. 5. Tanako, Kyoto Univers, Ty, JAPAN L. orory, Kroto Univers,tY, JAPAN

Drot. Noburuk, inoue, University of TOKYO, APAN S. Nor , JAER!, JAPAN

4. Jeong, L i brarian, Korma havonced Energy Res ingt, KOREA Prot. D.I. Choi, The Korea Mdr. Inst of Se I \& Teen, ROREa Prel. B.S. i iley, University of Waikato, NEW ZEALAND institute of Plage Pnysies, PEOPLE's REPUBLIC of CHINA Librarian, institute of Phys., PEOLE's REPUALIC of ONIN

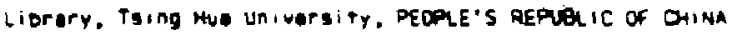
2, Li, Soutnwest inst. Pnysics. PEOPLE's RePUELIC OF CHINA Prof. J.A.C. Cagrel, inst Suderior Penico, Roprugal Or. Ostovion Perrus, al I Cuza unimarsity. Romania Or. Jea da villiars. Fusion Studies. AEC, SO AFRICA Prof. M.A. Wol Iberg. University of Notal, So afRich C.I.E.M.A.T. Fusion Div. Liorary, SPAIN Dr. Lennart Stentio, University of UNEA, SWEDEN Liorary, Rera: Institute of Teen, SLEDEN Prot. Hars wi ihelecon, Chalnars Univ of Tech, SWEDEN Cantre Phrs des PIassa, Ecole Polrtech Fed, SWI TZEALARD Bibliotheik, Fqu-Inst Vear Plage-Fysica, THE NETHERLANDS Mria Durgut, Midale East Technical University, TuRoky

Dr. 0.0. Rrutor, siberion Acad Sci, USSR

Dr. G.A. EI isenv, Kurchatov Insti rute, USSR Dr. V.A. Glumikn, Inst Electrophysicel Adporotus. J55A Prof. 0.5. Padienenxo, Inst, of Phys. \& Teen. US5R Dr. L.M. Kovrizhnykn, institute of Gon. Physics, USSR Nuclear Res. Establisment, Julich Ltd., W. GERAANY Bioligthek, Inst. Fur PIasmatorsenung. W. GERuAy Or. K. Sehindler, Ruhr-universitat Eochum, w. GERMant

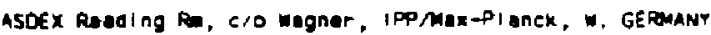
Liorarion, Maxtianck instirut. GeFahy Prof, A.K. Janter, inst of Phrs, rugoslavia 